International Journal of Pure and Applied Mathematics

Volume 95 No. 4 2014, 597-610

ISSN: 1311-8080 (printed version); ISSN: 1314-3395 (on-line version)

url: http://www.ijpam.eu

doi: http://dx.doi.org/10.12732/ijpam.v95i4.13

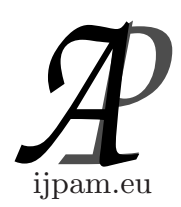

\title{
STRUCTURE OF NEAR-RINGS SATISFYING CERTAIN POLYNOMIAL IDENTITIES
}

\author{
Abdelkarim Boua $^{1 \S}$, Ahmed A.M. Kamal ${ }^{2}$ \\ ${ }^{1}$ Department of Mathematics \\ Faculty of Sciences of Agadir \\ Ibn Zohr University \\ P.O. Box 8106, Agadir, MOROCCO \\ ${ }^{2}$ Department of Mathematics \\ Faculty of Sciences \\ Cairo University, Giza, EGYPT \\ ${ }^{2}$ King Saud University \\ College of Science \\ Department of Mathematics \\ P.O. Box 2455, Riyadh, 11451, KINGDOM OF SAUDI ARABIA
}

\begin{abstract}
In this paper, we will introduce the concept of two-sided $\alpha$ generalized derivation in prime near-rings as it was outlined by the author N. Argac in [1]. Thereafter, we will generalize the same results proved by many authors (see [2], [4] and [5]) in the case of derivations, semiderivations and generalized derivations. Furthermore, we will give examples to demonstrate that the restrictions imposed on the hypothesis of various results are not superfluous.
\end{abstract}

AMS Subject Classification: 16N60, 16W25, 16Y30

Key Words: prime near-ring, $(\alpha, 1)$-derivation, $(1, \alpha)$-derivation, two-sided $\alpha$-derivation, two-sided $\alpha$-generalized derivation

Received: June 25, 2014

(C) 2014 Academic Publications, Ltd. url: www.acadpubl.eu

${ }^{\S}$ Correspondence author 


\section{Introduction}

A right near-ring is a set $N$ with two operations + and . such that $(N,+)$ is a group (not necessarily abelian) and $(N,$.$) is a semigroup satisfying the$ right distributive law $(x+y) z=x . z+y . z$ for all $x, y, z \in N$. A right nearring $N$ is called zero symmetric if $x .0=0$ for all $x \in N$ (recalling that A right distributivity yields $0 . x=0$. A near-ring $N$ is said to be prime if for all $x, y \in N, x N y=\{0\}$ implies $x=0$ or $y=0$. A nonempty subset $I$ of $N$ will be called a semigroup ideal if $I N \subseteq I$ and $N I \subseteq I$. A nonempty subset $I$ of $N$ is called stable by the additive law, if for any $x, y \in I, x+y \in I$ An additive mapping $\sigma: N \rightarrow N$ is called an involution if $\sigma(x y)=\sigma(y) \sigma(x)$ and $\sigma^{2}(x)=x$ for all $x, y \in N$. An additive mapping $d: N \longrightarrow N$ is said to be a derivation if $d(x y)=x d(y)+d(x) y$ for all $x, y \in N$. An additive mapping $F: N \longrightarrow N$ is said to be a generalized derivation on $N$ if there exists a derivation $d: N \longrightarrow N$ such that $F(x y)=F(x) y+x d(y)$ for all $x, y \in N$. An additive mapping $d: N \rightarrow N$ is called an $(\alpha, \beta)$-derivation if there exist functions $\alpha, \beta: N \rightarrow N$ such that $d(x y)=d(x) \alpha(y)+\beta(x) d(y)$ for all $x, y \in N$. An additive mapping $d: N \rightarrow N$ is called a two-sided $\alpha$-derivation if $d$ is an $(\alpha, 1)$-derivation as well as $(1, \alpha)$-derivation.

Now we introduce the notion of two-sided $\alpha$-generalized derivation of a near-ring $N$ as follows. An additive mapping $F: N \rightarrow N$ is called a $(\alpha, \beta)$-generalized derivation if there exist functions $\alpha, \beta: N \rightarrow N$ and a $(\alpha, \beta)$-derivation such that $F(x y)=F(x) \alpha(y)+\beta(x) d(y)$ for all $x, y \in N$. An additive mapping $F: N \rightarrow N$ is called a two-sided $\alpha$-derivation if $F$ is an $(\alpha, 1)$-generalized derivation as well as $(1, \alpha)$-generalized derivation. For $\alpha=1$, a two-sided $\alpha$ derivation (resp. two-sided $\alpha$-generalized derivation) is of course just a derivation (resp. generalized derivation). Recently, many authors have studied commutativity of prime and semiprime near-rings admitting suitably constrained additive mappings, as automorphisms, derivations, skew derivations, and generalized derivations acting on appropriate subsets of the near-rings. In the present paper, we would like to study the structure of a prime near-ring having a $(\sigma, 1)$-generalized derivation where $\sigma$ is an involution or a two-sided $\alpha$ generalized derivation where $\alpha$ is an homomorphism which satisfies suitable algebraic properties on semigroup ideals of $N$. 


\section{Some Preliminaries}

We begin with several lemmas, most of which are known. Those for which neither a proof nor a precise citation is given are to be found in [3], [4] and [6]. Unless it is stated otherwise, it will be assumed that $N$ is a zero-symmetric near ring and $d$ is a two-sided $\alpha$-derivation of $N$ associated with an endomorphism $\alpha$.

Lemma 1. [3, Lemma 1.3(1), Lemma 1.5 and Lemma 1.4(3)] Let $N$ be a prime near-ring and $I$ be a nonzero semigroup ideal of $N$.

(1) If $I a=\{0\}$ and $a \in N$, then $a=0$.

(2) If $Z(N)$ contains a nonzero semigroup ideal $I$, then $N$ is a commutative ring.

(3) If $x, y \in N$ and $x I y=\{0\}$, then $x=0$ or $y=0$.

Lemma 2. [6, Theorem 2] Let $N$ be a prime near-ring and $I$ be a nonzero semigroup ideal of $N$. If I stable by the additive law and admits an involution $\sigma$, then $N$ is a ring.

Lemma 3. Let $N$ be a prime near-ring. If $N$ admits a two-sided $\alpha$ derivation $d$, then $d(x) \alpha(y)+x d(y)=x d(y)+d(x) \alpha(y)$ for all $x, y \in N$.

Proof. The proof is the same of the proof of Proposition 1 of [7].

Lemma 4. Let $N$ be a prime near-ring and $d$ is a two-sided $\alpha$-derivation where $\alpha$ is an homomorphism, then

(1) $x(d(y) \alpha(z)+y d(z))=x d(y) \alpha(z)+x y d(z)$ for all $x, y, z \in N$.

(2) If $a d(x)=0$ for all $x \in N$ and $a \in N$, then $a=0$ or $d=0$.

Proof. (1) From the sample calculation of $d((x y) z)=d(x(y z))$, we obtain the required result.

(2) Assume that $a d(x)=0$ for $a \in N$ and for all $x \in N$. According to lemma 3 , we have

$$
\begin{aligned}
0 & =a d(x y) \\
& =a(d(x) \alpha(y)+x d(y)) \\
& =a d(x) \alpha(y)+a x d(y) \\
& =\operatorname{axd}(y) \text { for all } x, y \in N .
\end{aligned}
$$


Hence, $a N d(y)=\{0\}$ for all $x, y \in N$. By primeness of $N$, we conclude that $a=0$ or $d=0$, whereby the lemma is proved.

The following lemma is very useful in the sequel and it is a special case of Theorem 1 and Theorem 3 of [4].

Lemma 5. Let $N$ be a prime near-ring admitting a generalized derivation $F$ associated with a nonzero derivation $d$.

(1) $[4$, Theorem 1] If $F([x, y])=0$ for all $x, y \in N$, then $N$ is a commutative ring.

(2) If $F(x \circ y)=0$ for all $x, y \in N$, then $N$ is a commutative ring of characteristic 2 .

Proof. (2) The proof of $N$ is a commutative ring is the same as of the proof of Theorem 3 of [4]. For all $x, y \in N, F(x \circ y)=0$ implies that $F(x y)+F(x y)=0$ for all $x, y \in N$. Replacing $y$ by $y z, z \in N$, we get

$$
\begin{aligned}
0 & =F(x y z)+F(x y z) \\
& =F(x y) z+x y d(z)+F(x y) z+x y d(z) \\
& =(F(x y)+F(x y)) z+(2 x) y d(z) \\
& =(2 x) y d(z) \text { for all } x, y, z \in N .
\end{aligned}
$$

The primeness implies that $2 N=\{0\}$.

Lemma 6. [3, Lemma 1.2(i), (ii)] Let $N$ be a prime near-ring and $z \in$ $Z(N)-\{0\}$. Then $z$ is not a zero divisor. Moreover, if $z+z \in Z(N)$, then $(N,+)$ is abelian.

We conclude with the following useful remark.

Remark 1. Let $N$ be a prime near-ring admitting a two-sided $\alpha$-generalized derivation $F$ associated with a two-sided $\alpha$-derivation $d$. If $d \neq 0$, then $F \neq 0$. So it is sufficient to write $d \neq 0$ for both cases $(d \neq 0$ and $F \neq 0)$.

\section{Some Results Involving $(\sigma, 1)$-Generalized Derivations}

This Section is devoted to studying the structure of a zero symmetric prime right near-ring $N$ admitting a nonzero $(\sigma, 1)$-generalized derivation $F$ associated with a $(\sigma, 1)$-derivation $d$ where $\sigma$ is an involution. More precisely, we will prove the following. 
Theorem 1. Let $N$ be a prime near-ring and $I$ be a nonzero semigroup ideal of $N$ stable by the additive law. If $I$ admits a nonzero $(\sigma, 1)$-generalized derivation where $\sigma$ is an involution of $I$, then $N$ is a commutative ring.

Proof. By the hypothesis given and by Lemma 2, we get $N$ is a ring. So

$$
\begin{aligned}
d(x(y z)) & =d(x) \sigma(y z)+x d(y z) \\
& =d(x) \sigma(z) \sigma(y)+x d(y) \sigma(z)+x y d(z) \text { for all } x, y, z \in I .
\end{aligned}
$$

In another way

$$
\begin{aligned}
d((x y) z) & =d(x y) \sigma(z)+x y d(z) \\
& =(d(x) \sigma(y)+x d(y)) \sigma(z)+x y d(z) \\
& =d(x) \sigma(y) \sigma(z)+x d(y) \sigma(z)+x y d(z) \text { for all } x, y, z \in I .
\end{aligned}
$$

Comparing the two last expressions, we obtain

$$
d(x) \sigma(y) \sigma(z)=d(x) \sigma(z) \sigma(y) \text { for all } x, y, z \in I .
$$

By using $N$ is a ring, the equation (1) can be rewritten as

$$
d(x)[\sigma(y), \sigma(z)]=0 \text { for all } x, y, z \in I .
$$

By definition of $F$ and the same techniques introduced previously, we arrive at

$$
F(x)[\sigma(y), \sigma(z)]=0 \text { for all } x, y, z \in I .
$$

Taking $t x$ instead of $x$ in (3), we have

$$
F(t) \sigma(x)[\sigma(y), \sigma(z)]+t d(x)[\sigma(y), \sigma(z)]=0 \quad \text { for all } x, y, z, t \in I .
$$

Using (2), then (4) becomes

$$
F(t) \sigma(x)[\sigma(y), \sigma(z)]=0 \text { for all } x, y, z, t \in I .
$$

Applying $\sigma$ again, we get

$$
[z, y] x \sigma(F(t))=0 \text { for all } x, y, z, t \in I .
$$

This means that

$$
[z, y] I \sigma(F(t))=\{0\} \text { for all } y, z, t \in I .
$$

Using Lemma $1(3), \sigma$ is an involution and $F \neq 0$, the above expression becomes $[z, y]=0$ for all $y, z \in I$. Substituting $y t$ for $y$ where $t \in N$. The upshot is that $y[z, t]=0$ for all $y, z \in I, t \in N$, which implies that $I[z, t]=0$ for all $z \in I, t \in N$. From Lemma 1(1) and Lemma 1(2), we conclude that $N$ is a commutative ring. 
Corollary 1. Let $N$ be a prime near-ring. If $N$ admits a nonzero $(\sigma, 1)$ generalized derivation where $\sigma$ is an involution of $N$, then $N$ is a commutative ring.

Corollary 2. Let $N$ be a prime near-ring and $\sigma$ is an involution of $N$. If $N$ admits a nonzero $(\sigma, 1)$-derivation, then $N$ is a commutative ring.

Example 1. Let $N$ be the noncommutative prime $\operatorname{ring} M_{2}\left(\mathbb{Z}_{2}\right)$ and $d$ be the inner derivation induced by the element $\left[\begin{array}{ll}0 & 1 \\ 0 & 0\end{array}\right]$. Clearly that $d$ is a nonzero $(1,1)$-derivation associated with $\sigma=1$. Therefore, the condition " $\sigma$ is an involution" in Theorem 1 is not superfluous.

The following example shows the necessity of the primeness in Theorem 1.

Example 2. Let $S$ be a non-abelian right near-ring. We define $N, I$ and $\sigma: N \rightarrow N$ by:

$$
\begin{gathered}
N=I=\left\{\left(\begin{array}{lll}
0 & 0 & x \\
0 & 0 & y \\
0 & 0 & 0
\end{array}\right) \mid x, y \in S\right\}, \sigma\left(\begin{array}{lll}
0 & 0 & x \\
0 & 0 & y \\
0 & 0 & 0
\end{array}\right)=\left(\begin{array}{lll}
0 & 0 & y \\
0 & 0 & x \\
0 & 0 & 0
\end{array}\right) \\
F\left(\begin{array}{lll}
0 & 0 & x \\
0 & 0 & y \\
0 & 0 & 0
\end{array}\right)=\left(\begin{array}{lll}
0 & 0 & 0 \\
0 & 0 & y \\
0 & 0 & 0
\end{array}\right), d\left(\begin{array}{lll}
0 & 0 & x \\
0 & 0 & y \\
0 & 0 & 0
\end{array}\right)=\left(\begin{array}{lll}
0 & 0 & x \\
0 & 0 & 0 \\
0 & 0 & 0
\end{array}\right) .
\end{gathered}
$$

It is easy to see that $N$ is not prime near-ring and $\sigma$ is an involution of $N$. Also, it is clear that $F$ is a $(\sigma, 1)$-generalized derivation associated with a $(\sigma, 1)$ derivation $d$, but $N$ is not a ring.

\section{Two-Sided $\alpha$-Generalized Derivations Acting on Prime Near-Rings}

In this section, $F$ will denote a two-sided $\alpha$-generalized derivation associated with a nonzero two-sided $\alpha$-derivation $d$, where $\alpha$ is an endomorphism of $N$. The main purpose of this paragraph is to generalize several results du to some authors (see [2], [4] and [5]) concerning the study of commutativity of prime right near-ring satisfying certain differential identities. For more details see the following results. 
Theorem 2. Let $N$ be a prime near-ring. If $N$ admits a two-sided $\alpha$ generalized derivation $F$ associated with a nonzero two-sided $\alpha$-derivation $d$, where $\alpha$ is an endomorphism of $N$, then the following statements are equivalent:

(1) $F([u, v])=0$ for all $u, v \in N$,

(2) $N$ is a commutative ring.

Proof. It is obvious that (2) implies (1).

$(1) \Rightarrow(2)$ Suppose that

$$
F([u, v])=0 \text { for all } u, v \in N .
$$

By definition of $F$, we get

$$
F(u) v+\alpha(u) d(v)=F(u) \alpha(v)+u d(v) \text { for all } u, v \in N .
$$

Replacing $u$ by $[x, y]$ in (8) and applying (7), we obtain

$$
\alpha([x, y]) d(v)=[x, y] d(v) \text { for all } x, y, v \in N,
$$

or equivalently,

$$
(\alpha([x, y])-[x, y]) d(v)=0 \text { for all } x, y, v \in N .
$$

Since $d \neq 0$, according to Lemma 4(2), (9) implies that

$$
\alpha([x, y])=[x, y] \text { for all } x, y \in N .
$$

Taking $[x, y]$ instead of $v$ in (8) and invoking (10), we arrive at

$$
\alpha(u) d([x, y])=u d([x, y]) \text { for all } x, y, u \in N .
$$

Since $\alpha$ is an endomorphism of $N$. Replacing $u$ by $u t$ in (11) and using the above argument, we arrive at

$$
\alpha(u) t d([x, y])=u t d([x, y]) \text { for all } x, t, y, u \in N
$$

which leads to

$$
(\alpha(u)-u) N d([x, y])=\{0\} \text { for all } x, y, u \in N .
$$

Since $N$ is prime, then (12) implies

$$
\alpha=i d_{N} \text { or } d([x, y])=0 \text { for all } x, y \in N .
$$


(a) If $\alpha=i d_{N}$, then $F$ becomes a generalized derivation, in this case, Lemma 5 (1) assures that $N$ is a commutative ring.

(b) If $d([u, v])=0$ for all $u, v \in N$. Replacing $u$ by $u v$, it is obvious to see that

$$
\begin{aligned}
0 & =d([u v, v]) \\
& =d([u, v] v) \\
& =d([u, v]) \alpha(v)+[u, v] d(v) \\
& =[u, v] d(v) \text { for all } u, v \in N
\end{aligned}
$$

Thus,

$$
[u, v] d(v)=0 \text { for all } u, v \in N
$$

substituting $u r$ for $u$ in (14) and using (14), we arrive at

$$
[u, v] r d(v)=0 \text { for all } r, u, v \in N
$$

which implies that

$$
[u, v] N d(v)=\{0\} \text { for all } u, v \in N
$$

By 3-primeness of $N$, we find that

$$
v \in Z(N) \text { or } d(v)=0 \text { for all } v \in N \text {. }
$$

If there is an element $v_{0} \in N$ such that $v_{0} \in Z(N)$, then by Lemma 3 , we have

$$
\begin{aligned}
d\left(v_{0}\right) \alpha(u)+v_{0} d(u) & =d\left(v_{0} u\right) \\
& =d\left(u v_{0}\right) \\
& =\alpha(u) d\left(v_{0}\right)+d(u) v_{0} \text { for all } u \in N
\end{aligned}
$$

the upshot is reduced to $d\left(v_{0}\right) \alpha(u)=\alpha(u) d\left(v_{0}\right)$ for all $u \in N$. On the other hand, if $v \notin Z(N)$, then equation (15) implies $d(v)=0$ which shows that $d(v) \alpha(u)=\alpha(u) d(v)$ for all $u \in N$. So we have the following equation

$$
d(v) \alpha(u)=\alpha(u) d(v) \text { for all } u, v \in N .
$$

Since

$$
\begin{aligned}
d(u) v+\alpha(u) d(v) & =d(u v) \\
& =d(v u) \\
& =v d(u)+d(v) \alpha(u) \text { for all } u, v \in N
\end{aligned}
$$


by (16), the above expression gives

$$
d(u) v=v d(u) \text { for all } u, v \in N .
$$

Replacing $u$ by $d(u) t$ in (17) and by application of (17), we arrive at

$$
d^{2}(u) \alpha(t) v=v d^{2}(u) \alpha(t) \text { for all } u, v, t \in N .
$$

Expanding the last equation and using (17) again, we get

$$
[\alpha(t), v] N d^{2}(u)=\{0\} \text { for all } u, v, t \in N .
$$

Since $N$ is prime, then (18) implies that

$$
d^{2}(u)=0 \text { or } \alpha(t) \in Z(N) \text { for all } u, t \in N .
$$

(1) If $d^{2}(u)=0$ for all $u \in N$. By definition of $d$, we have

$$
d(u) v+\alpha(u) d(v)=d(u) \alpha(v)+u d(v) \text { for all } u, v \in N .
$$

Replace $u$ by $d(u)$ in (20), to get

$$
\alpha(d(u)) d(v)=d(u) d(v) \text { for all } u, v \in N
$$

which reduces to

$$
(\alpha(d(u))-d(u)) d(v)=0 \text { for all } u, v \in N .
$$

Since $d \neq 0$, by application of Lemma 4(2), (21) gives $\alpha(d(u))=d(u)$ for all $u \in N$. In this case, putting $u v$ instead of $u$, we have

$$
\alpha(d(u) v+\alpha(u) d(v))=d(u) \alpha(v)+u d(v) \text { for all } u, v \in N
$$

this means that,

$$
d(u) \alpha(v)+\alpha^{2}(u) d(v)=d(u) \alpha(v)+u d(v) \text { for all } u, v \in N .
$$

And therefore,

$$
\left(\alpha^{2}(u)-u\right) d(v)=0 \text { for all } u, v \in N .
$$

By Lemma $4(2)$ and $d \neq 0$, we obtain $\alpha^{2}=i d_{N}$. In this case, replacing $u$ by $x \alpha(t)$ and $v$ by $x$ in (17), we get

$$
d(x) t x=x d(x) t \text { for all } x, t \in N
$$


which implies that

$$
[x, t] N d(x)=\{0\} \text { for all } x, t \in N \text {. }
$$

In view of the primeness of $N$, the above expression yields that

$$
d(x)=0 \text { or } x \in Z(N) \text { for all } x \in N .
$$

If there exists an element $x_{0} \in N$ such that $x_{0} \in Z(N)$, then replacing $u$ by $x_{0} \alpha(t)$ in (17), we arrive at

$$
[v, t] N d\left(x_{0}\right)=\{0\} \text { for all } v, t \in N .
$$

By the primeness of $N$, the last expression gives

$$
d\left(x_{0}\right)=0 \text { or } v \in Z(N) \text { for all } v \in N .
$$

Since $d \neq 0$, by (23) and (24) we arrive at $N \subseteq Z(N)$, according to Lemma 1 (2), we conclude that $N$ is a commutative ring.

(2) If $\alpha(t) \in Z(N)$. Substituting $u t$ for $u$ in (17), we obtain

$$
d(u) t v+\alpha(u) d(t) v=v d(u) t+v \alpha(u) d(t) \text { for all } u, v, t \in N
$$

which is reduced to

$$
[v, t] N d(u)=\{0\} \text { for all } u, v, t \in N .
$$

By the primeness of $N$ and $d \neq 0$, we arrive at $N \subseteq Z(N)$ and by Lemma 1(2), we find that $N$ is a commutative ring, which complete the required proof.

If we replace the product $[x, y]$ by $x \circ y$ in Theorem 2 , then $N$ is a commutative ring of characteristic 2 . In fact, we obtain the following result:

Theorem 3. Let $N$ be a prime near-ring. If $N$ admits a two-sided $\alpha$ generalized derivation $F$ associated with a nonzero two-sided $\alpha$-derivation $d$, where $\alpha$ is a surjective endomorphism of $N$, such that $F(u \circ v)=0$ for all $u, v \in N$, then $N$ is a commutative ring of characteristic 2 .

Proof. Assume that

$$
F(u \circ v)=0 \text { for all } u, v \in N .
$$

Replacing $u$ by $x \circ y$ in (8) and from (25) it follows that

$$
(\alpha(x \circ y)-(x \circ y)) d(v)=0 \text { for all } x, y, v \in N \text {. }
$$


By Lemma $4(2)$ and $d \neq 0$, we get

$$
\alpha(x \circ y)=x \circ y \text { for all } x, y \in N .
$$

Substituting $x \circ y$ for $v$ in (18) and using (27), we obtain

$$
\alpha(u) d(x \circ y)=u d(x \circ y) \text { for all } x, y, u \in N .
$$

Taking ut instead of $x$ in (28) and by application of (28), we find that

$$
(\alpha(u)-u) N d(x \circ y)=\{0\} \text { for all } x, y, u \in N
$$

which, by virtue of the primeness of $N$, proves that

$$
\alpha=i d_{N} \text { or } d(x \circ y)=0 \text { for all } x, y \in N .
$$

(a) If $\alpha=i d_{N}$, then by Lemma 5(2) we get $N$ is a commutative ring of characteristic 2 .

(b) If $d(x \circ y)=0$ for all $x, y \in N$. Using the same techniques as we have introduced in the proof of precedent Theorem in the case where $[u, v]$ is replaced by $u \circ v$, one can easily see that

$$
u v d(v)=-v u d(v) \text { for all } u, v \in N .
$$

Taking $u t$ instead of $u$ in (30) and using (30), we find that

$$
(-v u+u v) N d(-v)=\{0\} \text { for all } u, v \in N .
$$

By the primeness on $N$, we get

$$
v \in Z(N) \text { or } d(v)=0 \text { for all } v \in N .
$$

(1) If there is an element $v \in N$ such that $v \in Z(N)$, then $d(v u)=d(u v)$ for all $u \in N$ which implies by using Lemma 3 that

$$
\begin{aligned}
d(v) \alpha(u)+v d(u) & =d(u) v+\alpha(u) d(v) \\
& =\alpha(u) d(v)+d(u) v \text { for all } u \in N .
\end{aligned}
$$

Thus, $v \in Z(N)$ implies $d(v) \alpha(u)=\alpha(u) d(v)$ for all $u \in N$. $\alpha$ is surjective implies $d(v) \in Z(N)$. Therefore, equation (32) becomes $d(v) \in Z(N)$ for all $v \in N$ which means that $d(N) \subseteq Z(N) . \quad d \neq 0$ implies the existence of an element $a \in N$ such that $d(a)$ is not a zero divisor element in $N$ by Lemma 6 . Using $d$ is $(\alpha, 1)$-derivation and Lemma 3 , we have

$$
d(x y)=d(x) \alpha(y)+x d(y)
$$




$$
=x d(y)+d(x) \alpha(y) \text { for all } x, y \in N \text {. }
$$

Multiplying $d(x y)$ by $\alpha(y)$ in the right and the left respectively and using $d(N) \subseteq Z(N)$, we get

$$
\begin{aligned}
d(x y) \alpha(y) & =(x d(y)+d(x) \alpha(y)) \alpha(y) \\
& =x d(y) \alpha(y)+d(x) \alpha(y) \alpha(y) \\
& =x \alpha(y) d(y)+\alpha(y) \alpha(y) d(x)
\end{aligned}
$$

By using Lemma 3 and Lemma 4(1),

$$
\begin{aligned}
\alpha(y) d(x y) & =\alpha(y)(x d(y)+d(x) \alpha(y)) \\
& =\alpha(y) x d(y)+\alpha(y) d(x) \alpha(y) \\
& =\alpha(y) x d(y)+\alpha(y) \alpha(y) d(x) .
\end{aligned}
$$

So $x \alpha(y) d(y)=\alpha(y) x d(y)$, which means that $(x \alpha(y)-\alpha(y) x) d(y)=0$ for all $x, y \in N$. Since $d(a)$ is not a right zero divisor in $N$, we get

$$
x \alpha(a)=\alpha(a) x \text { for all } x \in N
$$

Again, multiplying $d(x y)$ by $\alpha(a)$ in the right and the left respectively and using equation (33), we have $(\alpha(a) \alpha(y)-\alpha(y) \alpha(a)) d(x)=0$ for all $x, y \in N$. Using $d(a)$ is not a right zero divisor in $N$, we get

$$
\alpha(a) \alpha(y)=\alpha(y) \alpha(a) \text { for all } y \in N \text {. }
$$

Now, multiplying $d(x a)$ by $\alpha(z)$ in the right and the left respectively and using equation (34), it follows that

$$
x \alpha(z)=\alpha(z) x \text { for all } x, z \in N \text {. }
$$

Since $\alpha$ is surjective, we conclude that $N$ is a commutative near-ring. We have $0 \neq d(a) \in Z(N)$ and $d(a)+d(a)=d(a+a) \in Z(N)$. Thus, $(N,+)$ is an abelian group by Lemma 6 . Therefore, $N$ is a commutative ring. The proof of $N$ is of characteristic 2 is the same as the proof of Lemma $5(2)$.

Example 3. Let $N=M_{2}\left(\mathbb{Z}_{5}\right)$ and $d$ be the inner derivation induced by the element $\left[\begin{array}{ll}0 & 1 \\ 0 & 0\end{array}\right]$. Then $N$ is a non-commutative prime ring and $d\left(\left[\begin{array}{ll}a & b \\ c & d\end{array}\right]\right)=$ $\left[\begin{array}{cc}c & d-a \\ 0 & -c\end{array}\right]$. Let $x=\left[\begin{array}{ll}0 & 0 \\ 1 & 0\end{array}\right]$ and $y=\left[\begin{array}{ll}1 & 0 \\ 0 & 0\end{array}\right]$. Then $[x, y]=x \circ y=$ 
$\left[\begin{array}{ll}0 & 0 \\ 1 & 0\end{array}\right]$ and $d([x, y])=d(x \circ y)=\left[\begin{array}{cc}1 & 0 \\ 0 & -1\end{array}\right] \neq\left[\begin{array}{ll}0 & 0 \\ 0 & 0\end{array}\right]$. If we take $\alpha=i d_{N}$ the identity automorphism of $N$, then $d$ is a nonzero two-sided $\alpha$-generalized derivation associated with a nonzero two-sided $\alpha$-derivation $d$. Therefore, this example shows that the condition " $F([u, v])=0$ for all $u, v \in N$ " in Theorem 2 and the condition " $F(u \circ v)=0$ for all $u, v \in N$ " in Theorem 3 are not superfluous.

The following example shows that the primeness hypothesis in Theorem 2 and Theorem 3 cannot be omitted.

Example 4. Let $S$ be a non-abelian right near-ring and

$$
N=\left\{\left(\begin{array}{lll}
0 & x & y \\
0 & 0 & 0 \\
0 & 0 & 0
\end{array}\right)^{\mid x, y \in S}\right\}
$$

Defining the maps $F, d, \alpha: N \rightarrow N$ as the following:

$$
\begin{aligned}
F\left(\begin{array}{lll}
0 & x & y \\
0 & 0 & 0 \\
0 & 0 & 0
\end{array}\right)= & \left(\begin{array}{lll}
0 & x & 0 \\
0 & 0 & 0 \\
0 & 0 & 0
\end{array}\right), d\left(\begin{array}{lll}
0 & x & y \\
0 & 0 & 0 \\
0 & 0 & 0
\end{array}\right)=\left(\begin{array}{lll}
0 & 0 & y \\
0 & 0 & 0 \\
0 & 0 & 0
\end{array}\right), \\
& \alpha\left(\begin{array}{lll}
0 & x & y \\
0 & 0 & 0 \\
0 & 0 & 0
\end{array}\right)=\left(\begin{array}{lll}
0 & y & 2 x \\
0 & 0 & 0 \\
0 & 0 & 0
\end{array}\right) .
\end{aligned}
$$

Then, it is clear that $N$ is not prime right near-ring admitting a non-zero two-sided $\alpha$-generalized derivation $F$ associated with a nonzero two-sided $\alpha$ derivation. Moreover, it is easy to verify that $F$ satisfy the properties:

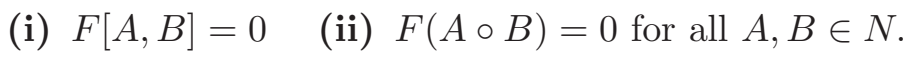

However, $N$ is not a commutative ring.

\section{References}

[1] Argac, N.: On near-rings with two-sided $\alpha$-derivations. Turk. J. Math., 28 (2004), 195-204.

[2] Ashraf, M; Shakir, A.: On $(\sigma, \tau)$-derivations of prime near-rings-II, Sarajevo J. Math., 4 (16) (2008) 23-30. 
[3] Bell, H. E.: On derivations in near-rings. II. Nearrings, nearfields and $K$ loops (Hamburg, 1995), 191-197, Math. Appl., 426, Kluwer Acad. Publ., Dordrecht, 1997. DOI: 10.1007/978-94-009-1481-0_10.

[4] Boua, A.; Oukhtite, L. and Bell, H. E.: Differential identities on semigroup ideals of right near-rings. Asian-Eur. J. Math. 6 (4) (2013), 8pp. DOI: 10.1142/S1793557113500502

[5] Boua, A.; Oukhtite, L.: Semiderivations satisfying certain algebraic identities on prime near-rings, Asian Eur. J. Math., 6 (3) (2013), 1-8. DOI: 10.1142/S1793557113500435.

[6] Boua, A.; Raji, A.: 3-prime near-rings with involutions, Int. J. Math. Eng. Sci., 2 (8) (2013), 12-16.

[7] Wang, X. K.: Derivations in prime near-rings. Proc. Amer. Math. Soc., 121 (2), 1994, 361-366. DOI : 10.1090/s0002-9939-1994-1181177-7. 